\title{
Theoretical description of adiabatic laser alignment and mixed-field orientation: the need for a non-adiabatic model
}

\author{
J. J. Omiste, ${ }^{1}$ M. Gärttner, ${ }^{2}$ P. Schmelcher, ${ }^{3}$ R. González-Férez, ${ }^{1}$ L. \\ Holmegaard, ${ }^{4,5}$ J. H. Nielsen, ${ }^{6}$ H. Stapelfeldt, ${ }^{4,7}$ and J. Küpper ${ }^{5,8,9}$ \\ ${ }^{1}$ Instituto Carlos I de Física Teórica y Computacional, and Departamento de Fúsica Atómica, \\ Molecular y Nuclear, Universidad de Granada, 10871 Spain \\ ${ }^{2}$ Max Planck Institut für Kernphysik, Saupfercheckweg 1, 69117 Heidelberg, Germany. \\ ${ }^{3}$ Zentrum für Optische Quantentechnologien, Universität Hamburg, \\ Luruper Chaussee 149, Hamburg, 22761, Germany. \\ ${ }^{4}$ Department of Chemistry, University of Aarhus, 8000 Aarhus C, Denmark. \\ ${ }^{5}$ Center for Free-Electron Laser Science, DESY, Notkestrasse 85, 22607 Hamburg, Germany \\ ${ }^{6}$ Department of Physics and Astronomy, University of Aarhus, 8000 Aarhus C, Denmark. \\ ${ }^{7}$ Interdisciplinary Nanoscience Center (iNANO), University of Aarhus, 8000 Aarhus C, Denmark. \\ ${ }^{8}$ Fritz-Haber-Institut der MPG, Faradayweg 4-6, 14195 Berlin, Germany \\ ${ }^{9}$ University of Hamburg, Luruper Chaussee 149, 22761 Hamburg, Germany
}

(Dated: September 6, 2018)

\begin{abstract}
We present a theoretical study of recent laser-alignment and mixed-field-orientation experiments of asymmetric top molecules. In these experiments, pendular states were created using linearly polarized strong ac electric fields from pulsed lasers in combination with weak electrostatic fields. We compare the outcome of our calculations with experimental results obtained for the prototypical large molecule benzonitrile $\left(\mathrm{C}_{7} \mathrm{H}_{5} \mathrm{~N}\right)$ [J. L. Hansen et al, Phys. Rev. A, 83, 023406 (2011)] and explore the directional properties of the molecular ensemble for several field configurations, i. e., for various field strengths and angles between ac and dc fields. For perpendicular fields one obtains pure alignment, which is well reproduced by the simulations. For tilted fields, we show that a fully adiabatic description of the process does not reproduce the experimentally observed orientation, and it is mandatory to use a diabatic model for population transfer between rotational states. We develop such a model and compare its outcome to the experimental data confirming the importance of non-adiabatic processes in the field-dressed molecular dynamics.
\end{abstract}

\section{INTRODUCTION}

Controlling molecular motions has direct impact in a wide variety of molecular sciences, including stereochemistry [1] [4], molecular-frame investigations of geometric and electronic properties, such as photoelectron angular distributions [5-7] and high-harmonic generation [8, 9], as well as for diffractive imaging of gasphase molecules [10, 11], aiming at recording the "molecular movie" [12]. Recently, there has been tremendous progress in the control of the translational [13] and rotational [14 17] motions of even complex molecules. For extremely well controlled ultracold alkali dimers, direct quantum effects in the stereodynamics of molecular reactions have recently been observed [18].

Angular confinement of molecular ensembles is referred to as alignment - the confinement of molecule-fixed axes along laboratory-fixed axes - and orientation - adding a well-defined direction. Traditionally, these two levels of angular control have been separated: strong ac fields from pulsed lasers have been used to create alignment [14], whereas state-selection [19] and brute-force orientation using strong dc electric fields [20, 21] have been used to create orientation (which typically also creates alignment). About a decade ago it was realized that strong simultaneous alignment and orientation could be created using combined ac and dc electric fields [22, 23]. This has been experimentally verified in a few cases 24
26]. Adding methods to control the translational motion one can perform a quantum-state selection before the alignment and orientation experiment 13, 27, 28]. This two-step approach has allowed the creation of unprecedented degrees of one-dimensional (1D) and threedimensional (3D) alignment and orientation even for complex asymmetric top molecules [6, 15, 29 31].

Theoretical studies of the rotational spectra in the presence of combined electrostatic and non-resonant radiative fields have been restricted to linear and symmetric top molecules so far [22, 23, 32]. Recently, some of us developed the theory for asymmetric top molecules in combined fields [33]. When the static and linearly polarized laser field directions are tilted with respect to one another, the symmetries of the corresponding Hamiltonian are significantly reduced. This is the most general field configuration, its theoretical treatment being most challenging. Since each external field interaction breaks different symmetries of the field-free Hamiltonian, the order in which the fields are turned on determines the evolution of the field-dressed rotational dynamics. The labels of the eigenstates at a certain field configuration obtained by adiabatic following depend on the path through parameter space, i. e., monodromy is observed. For linear and symmetric top molecules exposed to external fields, the phenomena of classical and quantum monodromy have been encountered in the corresponding dressed spectra [34 36]. The labeling procedure is also 
numerically very demanding due to the large amount of genuine and avoided crossings occurring between adjacent states as one of the field parameters is varied.

In the present work, we describe the recent experimental results obtained for the alignment and orientation of asymmetric top molecules in combined fields 6, 15, 29 31. In the framework of the rigid rotor approximation, we perform a full investigation of the field-dressed eigenstates for those field configurations considered in the experiment. The Schrödinger equation for the rotational/pendular states is solved separately for each irreducible representation by expanding the wave function in a basis with the correct symmetries. Our theoretical analysis also includes i) a diabatic approximation to account for population transfer through the avoided crossings as the laser intensity is varied; ii) the velocity distribution of the ions after the Coulomb explosion; and iii) a volume effect model to describe the fact that not all the molecules feel the same laser intensity because the orienting and the detection laser pulses have finite spatial intensity profiles. The field-dressed eigenfunctions are weighted with the known relative state populations in the molecular beam [29]. Then, we compute the angular probability density functions for different field configurations and their velocity-mapping images (VMIs). The recently performed alignment and orientation experiment for benzonitrile $\left(\mathrm{BN}, \mathrm{C}_{7} \mathrm{H}_{5} \mathrm{~N}\right)$ molecules [6, 31] provides us with experimental data that is very well suited to present and discuss our theoretical model in a comparative study. We numerically compute the alignment and orientation for an ensemble of quantum-state selected benzonitrile molecules, and compare our results to the experimental data. For comparison with a cold thermal ensemble - without state selection - we also provide the corresponding results for a benzonitrile sample at $1 \mathrm{~K}$. In particular, for perpendicular fields, we obtain good agreement between the computational and experimental results for the degree of alignment. For tilted fields, we show that a fully adiabatic description of the rotational/pendular dynamics cannot reproduce the experimental results for the mixed-field orientation of benzonitrile. When a diabatic model is implemented for the treatment of the avoided crossings, our theoretical study reproduces with reasonable accuracy the experimental degree of orientation. Hence, we demonstrate the impact of non-adiabatic processes on the field-dressed molecular dynamics. We have developed a general theoretical description of alignment and mixed-field orientation for asymmetric tops in long pulses of strong ac electric and weak dc fields.

The paper is organized as follows: In Sec. П the relevant experimental details are described. The theoretical model is presented in Sec. III, which includes the discussion of the rigid rotor Hamiltonian and its symmetries, the diabatic model to treat the avoided crossings, the screen projection of the 3D probability densities and the experimental observables. The theoretical results for the alignment and orientation of a beam of benzonitrile molecules are compared to the experimental data in Sec. IV] The conclusions and outlook are provided in Sec. [V]

\section{EXPERIMENTAL DETAILS}

A detailed description of the alignment and mixedfield orientation experiments is given elsewhere [6, 29, 31]. Briefly, a pulsed, cold molecular beam of benzonitrile molecules seeded in helium is expanded from an Even-Lavie valve into vacuum. The molecular beam is skimmed before entering a $15 \mathrm{~cm}$ long electrostatic deflector $41 \mathrm{~cm}$ downstream from the nozzle. The deflector disperses the molecules in the beam according to their effective dipole moments, creating a vertically varying distribution of quantum states in the probe region $77 \mathrm{~cm}$ downstream from the nozzle. Alignment and orientation is induced by the dc electric field of the VMI spectrometer and by a strong Nd:YAG laser pulse (10 ns, $1064 \mathrm{~nm}$ ) and probed using ion imaging of $\mathrm{CN}^{+}$fragments following Coulomb explosion of benzonitrile with a strong ultrashort Ti:Sapphire laser pulse (30 fs, $800 \mathrm{~nm}$, $\left.5.4 \times 10^{14} \mathrm{~W} / \mathrm{cm}^{2}\right)$. Alignment and orientation experiments were performed for the undeflected beam and for the quantum-state selected sample at a vertical height of $1.75 \mathrm{~mm}$; see Figure 2 of reference 31 for details.

\section{THEORETICAL MODEL}

An exact theoretical quantum description of the alignment and orientation experiments is very demanding, since it requires the solution of the corresponding timedependent Schrödinger equation for each state populated in the molecular ensemble. Instead, we retreat to a quasistatic description parametric in the field strength and angles, and solve the time-independent Schrödinger equation for several field configurations. A diabatic model, based on the field-free symmetries, is used to account for the population transfer through the avoided crossings encountered as the YAG pulse intensity is varied.

\section{A. The Hamiltonian}

We consider a nonresonant laser field of intensity $I$ and linearly polarized along the $Z_{L}$-axis of the laboratory fixed frame (LFF), $\left(X_{L}, Y_{L}, Z_{L}\right)$, and an homogeneous electrostatic field of strength $E_{S}$ contained in the $X_{L} Z_{L^{-}}$ plane and forming an angle $\beta$ with $Z_{L}$. The rigid rotor Hamiltonian of a polar asymmetric top molecule exposed to this field configuration is given by

$$
\begin{aligned}
H= & J_{X_{M}}^{2} B_{X_{M}}+J_{Y_{M}}^{2} B_{Y_{M}}+J_{Z_{M}}^{2} B_{Z_{M}}-E_{S} \mu \cos \theta_{S} \\
& -\frac{2 \pi I}{c}\left(\alpha^{Z_{M} X_{M}} \cos ^{2} \theta+\alpha^{Y_{M} X_{M}} \sin ^{2} \theta \sin ^{2} \chi\right),
\end{aligned}
$$

with $B_{X_{M}}, B_{Y_{M}}$, and $B_{Z_{M}}$ being the rotational constants. The molecule or body fixed frame (MFF) 
$\left(X_{M}, Y_{M}, Z_{M}\right)$ is defined so that the permanent electric dipole moment $\mu$ is parallel to the $Z_{M}$-axis. The LFF and the MFF are related by the Euler angles $(\phi, \theta, \chi) 37$. The polarizability tensor is diagonal in the MFF with components $\alpha_{i i}$ with $i=X_{M}, Y_{M}, Z_{M}$, and the interaction with the laser field depends on the polarizability anisotropies $\alpha^{j i}=\alpha_{j j}-\alpha_{i i}, i, j=X_{M}, Y_{M}, Z_{M}$. The angle between the static electric field and the molecular $Z_{M}$-axis is $\theta_{S}$ with $\cos \theta_{S}=\cos \beta \cos \theta+\sin \beta \sin \theta \cos \phi$. This study is restricted to molecules with the permanent dipole moment parallel to one of the principal axis of inertia, as it is the case for benzonitrile.

Let us shortly summarize the approximations and assumptions made in the derivation of the Hamiltonian (1). We perform a non-relativistic description within the framework of the rigid rotor approximation, assuming that the electronic and vibrational structures are not affected by the external fields. In addition, we presuppose that the laser is non-resonant and that the inverse of the oscillation frequency is much larger than the rotational period of the molecular system and pulse duration. Thus, we can average over the rapid oscillations, so that the interaction of this field with the molecular dipole moment is zero, and only the interaction with the polarizability is left. Additionally, since the YAG laser pulse duration is much larger than the time scale of the rotational dynamics, we assume that the alignment and orientation processes are, in principle, adiabatic, and we take the time profile of the pulse as a constant equal to 1 . The validity of this assumption will be discussed in detail through the paper. The spatial dependence of the laser intensity will be also taken into account by means of a volume effect model, see below.

The configuration of the fields determines the symmetries of the Hamiltonian (1). For the field-free case, they are given by the spatial group $\mathrm{SO}(3)$ and the molecular point group $\mathrm{D}_{2}-$ i. e., the Fourgroup $\mathrm{V}$ - consisting of the identity and the two-fold rotations $C_{2}^{i}$ around the MFF $i$-axis, with $i=X_{M}, Y_{M}$ and $Z_{M}$. The Schrödinger equation associated with the Hamiltonian (1) can not be solved analytically, and only the total angular momentum, $J$, and its projection onto the $Z_{L}$-axis of the laboratory fixed frame, $M$, are good quantum number, whereas $K$, the projection of $\mathbf{J}$ on the $Z_{M}$-axis of the molecular fixed frame, is not conserved. Since an external field defines a preferred direction in space, the symmetries of the corresponding Hamiltonian are reduced compared to the field-free case. Here we consider three fieldconfigurations. For a single static field parallel to the LFF- $Z_{L}$-axis, the symmetry operations are $C_{2}^{Z_{M}}$, any arbitrary rotation around the $Z_{L}$-axis and a reflection in any plane containing $Z_{L}$, thus, $M$ is still conserved. For a certain $|M|$, there are 4 irreducible representation, and for $M \neq 0$ the states with $M$ and $-M$ are degenerate. If the molecule is exposed to both fields, with the electric field being rotated away from the $Z_{L}$-axis, the azimuthal symmetry is lost and $M$ ceases to be a good quantum number. In the perpendicular case, $\beta=90^{\circ}$, the Hamiltonian (1) is invariant under $C_{2}^{Z_{M}}$, a rotation of $\pi$ around the $X_{L}$-axis, and the reflection $\sigma_{X_{L}} Z_{L}\left(X_{L} Z_{L}\right.$ is the plane containing the fields); in consequence there are 8 irreducible representations. For tilted fields with $\beta \neq 90^{\circ}$, two symmetries are left, $\sigma_{X_{L} Z_{L}}$ and $C_{2}^{Z_{M}}$, and the corresponding group has only 4 irreducible representations. For a detailed description and analysis of the symmetries for all possible situations, we refer the reader to the recent works 33,38 .

The field-dressed eigenstates of an asymmetric top are characterized by the avoided crossings appearing between levels of the same symmetry as one of the parameters of the field configuration, i.e., $E_{S}, I$, or $\beta$, is varied. For non-collinear fields, the small amount of irreducible representations implies an eigenstate diagram with a high degree of complexity due to the large number of avoided crossings. Furthermore, a small degree of asymmetry on the inertia tensor facilitates the appearance of avoided crossings on the corresponding field-dressed states [39]. For the correct analysis of the computational results, these avoided crossings should be distinguished from genuine ones taking place between levels of different symmetries. Hence, the time-independent Schrödinger equation associated to the Hamiltonian (1) is solved for each irreducible representation by expanding the wave function in a basis that respects the corresponding symmetries [33].

The field-free states are identified by the quantum labels $J_{K_{a} K_{c}} M$, with $J$ and $M$ being good quantum numbers, and $K_{a}$ and $K_{c}$ the projections of $J$ onto the $Z_{M^{-}}$ axis of the molecular fixed frame in the oblate and prolate limiting cases [40], respectively. For reasons of addressability, we will denote the field dressed states by means of these field-free labels indicating if an adiabatic or diabatic picture has been used.

To illustrate the molecular dynamics in the stateselection process using the electric deflector, we plot the Stark energies and expectation value $\langle\cos \theta\rangle \equiv$ $\left\langle J_{K_{a} K_{c}} M|\cos \theta| J_{K_{a} K_{c}} M\right\rangle$ of the populated states of benzonitrile in Figure 1.(a) and (b) as a function of the electrostatic field strength. This plot covers the range of electric field strengths present in the deflector. It includes 84 individual rotational states accounting for $98 \%$ of the population of the molecular ensemble. This level diagram shows a complex structure with both genuine and avoided crossings. In the weak electrostatic field regime, both high- and low-field seekers are encountered, whereas in the pendular limit, and, in particular, in the regime of interest for the state selection experiment, all these levels are high-field-seekers. For $E_{S}>50 \mathrm{kV} \mathrm{cm}^{-1}$, most of these populated states present a significant orientation with $\langle\cos \theta\rangle>0.5$, and $32 \%$ of them have a strong one with $\langle\cos \theta\rangle>0.8$. The avoided crossings have a strong impact on the character of the involved states, and $\langle\cos \theta\rangle$ suffers large variations over tiny ranges of electrostatic field strength, see Figure 1(b). Note that the dc field applied in the orientation experiment, $286 \mathrm{Vcm}^{-1}$, is very small on the scale of this figure. 


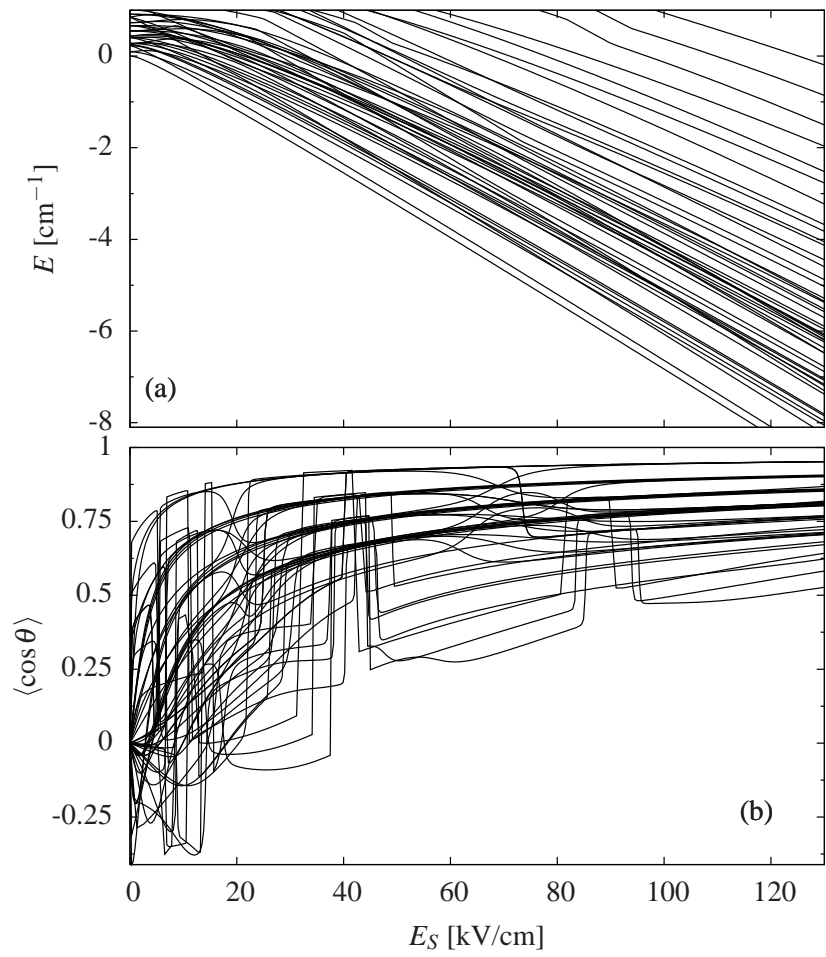

Fig. 1: (a) Energies and (b) expectation value $\langle\cos \theta\rangle$ as a function of the static field strength of the populated rotational states for benzonitrile.

\section{B. The diabatic model}

After entering the region of the extractor field, i.e., the static electric field of the VMI spetrometer, the molecules are exposed to a laser field with increasing intensity (YAG laser pulse). As the laser intensity varies, a certain state may undergo several avoided crossings with levels of the same symmetry. The presence of these avoided crossings as well as their diabatic or adiabatic nature have a strong impact on the outcome of the experiment.

Several theoretical studies have analyzed in detail the character of such avoided crossings for molecules exposed to an electrostatic field by using different adiabaticity criteria 39, 41 43]. Their main and common conclusion is that the assumption of a fully adiabatic dynamics is incorrect as no general statement can be made about the character of the avoided crossings. These works suggest that an investigation of the character of the avoided crossings encountered as $I$ is varied should be mandatory for a correct description of the experimental results. To do so, we use the following adiabatic passage criterion [44]

$$
\eta=\frac{\left\langle i\left|\frac{\partial H^{\prime}}{\partial t}\right| j\right\rangle}{\left(E_{i}-E_{j}\right)^{2}} \ll 1,
$$

where $E_{i}$ and $E_{j}$ are the eigenenergies for the states $i$ and $j$ and $H^{\prime}$ is the interaction term. Due to the large amount of avoided crossings in the laser field-dressed states, a systematic and detailed study of the adiabatic or diabatic character of all of them is unfeasible. Thus, we employ a simple diabatic model that provides an approximation to the dynamics, and determines the population transfer as the field parameters are varied. Note, that some previous works have proposed different population transfer models based on symmetry considerations [41, 42, 45].

If the fields are not collinear and the polarization of the laser pulse is parallel to the $Z_{L}$-axis, the electrostatic field induces the coupling of states with different field-free values of the quantum number $M$. For the experimentally used electrostatic field strength, $E_{S}=286 \mathrm{~V} \mathrm{~cm}^{-1}$, the Stark interaction is much weaker than the laser field one. Thus, the hybridization of the quantum number $M$ is so small that for a certain level $\left\langle M^{2}\right\rangle$ remains almost unperturbed and equal to its field free value. Our diabatic model consists in assuming that i) an avoided crossing between two levels with different field-free values of $M$ is considered as being crossed diabatically; and ii) crossings between levels with the same field-free value of $M$ are passed adiabatically. To illustrate the validity of this diabatic model we have analyzed two exemplary avoided crossings of the field-dressed spectrum with $\beta=45^{\circ}$ and $E_{S}=286 \mathrm{~V} \mathrm{~cm}^{-1}$ by means of the adiabatic passage parameter $\eta$ of equation (2). In this case, the interaction term is given by $H^{\prime}=(2 \pi I / c)\left(\alpha^{Z X} \cos ^{2} \theta+\right.$ $\left.\alpha^{Y X} \sin ^{2} \theta \sin ^{2} \chi\right)$. For the time profile of the YAG pulse, we use $I(t)=I \exp \left(-t^{2} /\left(2 \sigma^{2}\right)\right)$ with $I=5 \times 10^{11} \mathrm{~W} \mathrm{~cm}^{-2}$ and $\sigma=4.25$ ns (FWHM=10 ns). Figure 2(a), (d), (b), (e) and (c), (f) depict the energy, $\left\langle\cos ^{2} \theta\right\rangle$, and $\eta$ as a function of $I$ for the avoided crossings between the states $2_{02} 0$ and $2_{02} 1$, and $4_{04} 4$ and $3_{21} 1$, respectively. The levels $2_{02} 0$ and $2_{02} 1$ suffer an avoided crossing for $I \approx 3.5 \times 10^{9} \mathrm{~W} \mathrm{~cm}^{-2}$, with $\Delta E=5.5 \times 10^{-4} \mathrm{~cm}^{-1}$ and $\eta=192$. Hence, we conclude that it is crossed diabatically. In contrast, for the avoided crossing among the states $4_{04} 4$ and $3_{21} 1$ occurring at $I \approx 3.82 \times 10^{10} \mathrm{~W} \mathrm{~cm}^{-2}$, we obtain $\Delta E=8.69 \times 10^{-2} \mathrm{~cm}^{-1}$ and $\eta=0.12$, which according to equation (2) is an intermediate case - neither diabatic nor adiabatic. Using a fully adiabatic picture these two states are labeled $4_{04} 4$ and $3_{21} 1$, but using the diabatic model their labels are different since $\left\langle M^{2}\right\rangle=$ 3.999 and 4.007, respectively, for $I=3.6 \times 10^{10} \mathrm{~W} \mathrm{~cm}^{-2}$, which explains the mixing between both levels. The criterion (2) or the Landau-Zener formula do not classify this avoided crossing as being diabatic or adiabatic, but within our approximation we consider it to be adiabatic.

For tilted fields $\left(\beta \neq 90^{\circ}\right)$, a certain avoided crossings may involve two states one being oriented and the other one antioriented. Hence, considering it as diabatic or adiabatic has significant consequences on the final result for orientation. Of course, our diabatic model is an approximation, since many avoided crossings are encountered that do not fall clearly into the class of adiabatic or diabatic crossings according to our adiabatic passage criterion. Generally, these cases would require to solve the time-dependent Schrödinger equation including the time profile of the YAG pulse. 

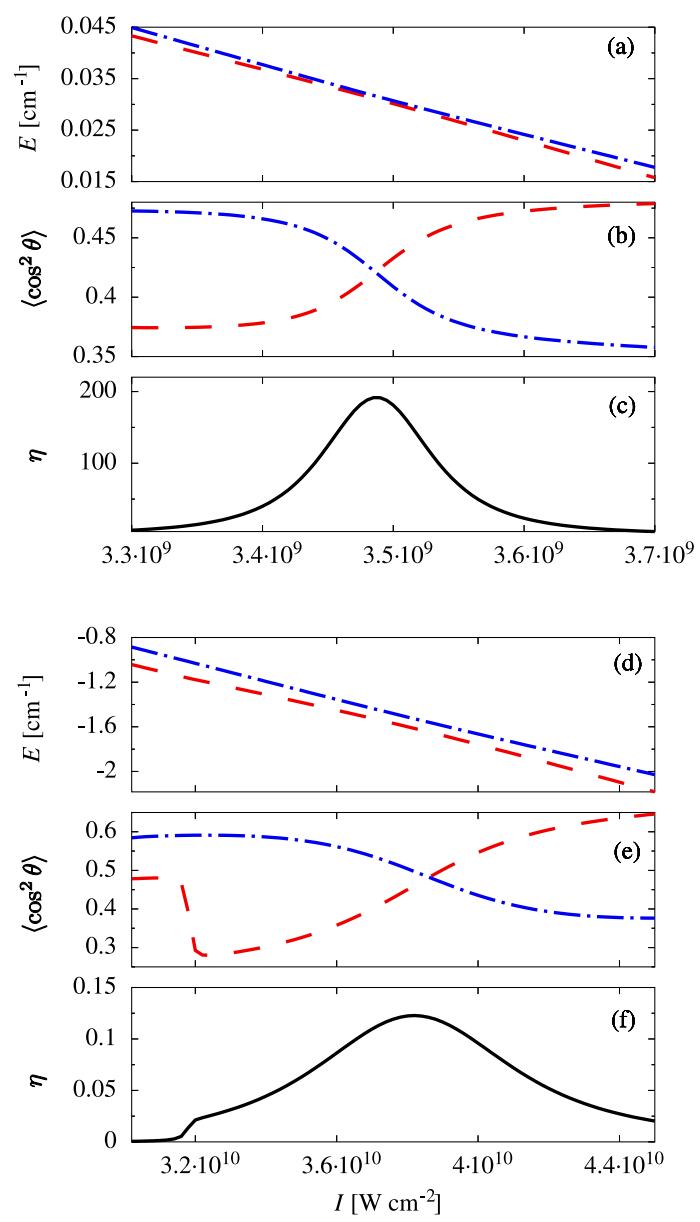

Fig. 2: Energy $E\left((\mathrm{a})\right.$ and (d)), expectation value $\left\langle\cos ^{2} \theta\right\rangle$ ((b) and (e)), adiabatic passage criterion parameter (2) ((c) and (f)) for the levels $2_{02} 0$ (dash-dotted line) and $2_{02} 1$ (dashed line) states; and for the states $4_{04} 4$ (dash-dotted line) and $3_{21} 1$ (dashed line) states as a function of the YAG laser intensity, for $E_{S}=286 \mathrm{~V} \mathrm{~cm}^{-1}$ and $\beta=45^{\circ}$. See the text for more details.

If the fields are perpendicular, the corresponding Hamiltonian (1) has 8 irreducible representations, and the amount of avoided crossings is significantly reduced compared to the $\beta \neq 90^{\circ}$ configuration. For the populated states of one irreducible respresentation, the expectation value $\left\langle\cos ^{2} \theta\right\rangle$ is plotted on Figure 3 as a function of the laser intensity, for $E_{S}=286 \mathrm{~V} \mathrm{~cm}^{-1}$ and $\beta=90^{\circ}$. The inset of this figure shows the energy levels in the strong-laser-field regime. For the laser intensities at which the experiment is performed, most of the populated levels are characterized by a pronounced alignment. In particular, we find $\left\langle\cos ^{2} \theta\right\rangle>0.8$ for all these states and $I>4.1 \times 10^{11} \mathrm{~W} \mathrm{~cm}^{-2}$. Since, the character - regarding alignment- of two states that had previously suffered an avoided crossing is very similar, passing through it diabatically or adiabatically is not crucial for the final alignment result.

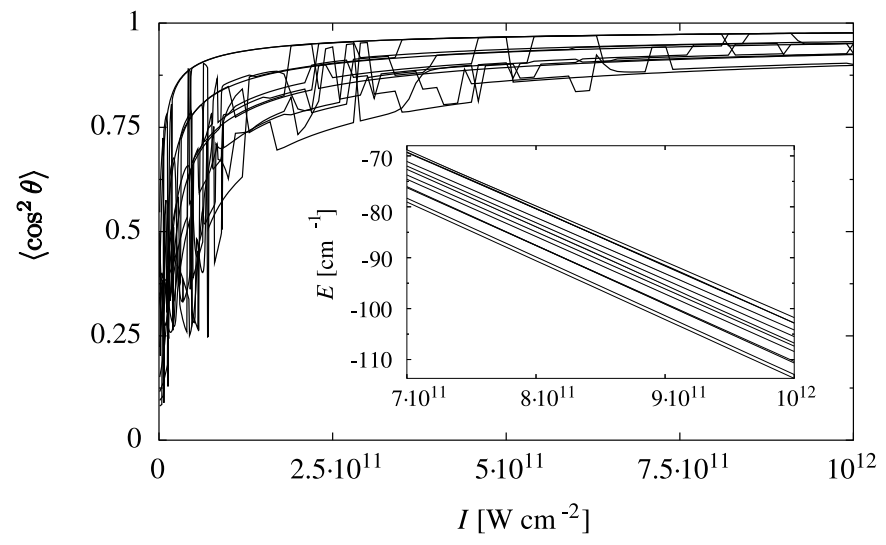

Fig. 3: Expectation value $\left\langle\cos ^{2} \theta\right\rangle$ and energies (inset panel) as a function of the YAG pulse intensity of the populated rotational states for benzonitrile, for $E_{S}=286 \mathrm{~V} \mathrm{~cm}^{-1}$ and $\beta=90^{\circ}$. Only one irreducible representation is presented.

\section{The projection of the probability density on the screen detector}

When a molecule is multiply ionized using an intense ultrashort laser pulse, it dissociates due to Coulomb repulsion and the created ionic fragments are collected in a 2D space resolving detector. Within the axial recoil approximation the momentum of the $\mathrm{CN}^{+}$fragments created in this process is parallel to the $\mathrm{C}-\mathrm{CN}$ bond direction before ionization. In order to calculate the screen image from the molecular wave functions we define a new reference frame $(x, y, z)$, containing the detector screen on its $y z$-plane. This frame is obtained by rotating the LFF by an angle $90^{\circ}-\beta$ about the $Y_{L}$-axis. Note that the electrostatic field is parallel to the $x$-axis, and the polarization vector of the linearly polarized YAG laser lies in the $x z$-plane, forming an angle $\beta$ with the $x$-axis.

An asymmetric top molecule in a certain state, characterized by the wavefunction $\Psi_{\gamma}(\theta, \phi, \chi)$, with $\gamma=$ $J_{K_{a} K_{c}} M$, is traveling towards a screen (velocity in the $x$-direction). Since the direction of the molecular dipole moment is independent of the angle $\chi$, the probability for the molecular $Z_{M}$-axis to be oriented according to $(\theta, \phi)$ is given by integrating in $\chi$ the absolute square of the wave function. The angular distribution $\rho_{\gamma}(\theta, \phi) d \Omega$ provides a measure of the amount of ions ejected into the solid angle $d \Omega=\sin \theta d \theta d \phi$. It is related to a spatial distribution on the $2 \mathrm{D}$ screen by $\rho_{\gamma}(y, z) d y d z=$ $\rho_{\gamma}(\theta(y, z), \phi(y, z))|J| \sin (\theta(y, z)) d y d z$, with $J$ being the Jacobian of the transformation between the coordinates $(\theta, \phi)$ and $(y, z)$. Assuming that all the ions have the same velocity, $v$ in absolute value, this transformation reads as

$$
\begin{aligned}
& y=a \sin \theta \sin \phi \\
& z=a(\cos \theta \sin \beta+\sin \theta \cos \phi \cos \beta),
\end{aligned}
$$

where $a=v t_{f}$, and $t_{f}$ is the time of flight to reach the screen. Note that with the definition (3) for the $y$ - 
coordinate, any point $(y, z)$ corresponds to two different orientations $(\theta, \phi)$ and $\left(\theta, 180^{\circ}-\phi\right)$. Thus, the screen image is the sum of two projection images, each obtained for a restricted range of $\phi$.

An important ingredient, that should be taken into account to obtain realistic screen images, is the alignment selectivity of the probe laser. We approximate the effectivity of the dissociation process by the factor $\cos ^{2} \alpha$, with $\alpha$ being the angle between probe laser polarization and the $\mathrm{C}-\mathrm{CN}$ bond direction [30]. Note that this approximation for modeling the probe selectivity as $\cos ^{2} \alpha$ is in accord with the experimental observations. Thus, the $2 \mathrm{D}$ screen spatial distribution is given by

$$
\rho_{\gamma}^{i}(y, z)=\frac{\rho_{\gamma}(\theta(y, z), \phi(y, z))}{a \sqrt{a^{2}-y^{2}-z^{2}}} A_{i}(y, z)
$$

where $A_{i}(y, z)$ is the mentioned aligment selectivity factor, and the index $i$ indicates the probe pulse polarization. For a linearly polarized probe beam in the $x$-direction, we obtain $A_{l}(y, z)=1-y^{2} / a^{2}-z^{2} / a^{2}$, and in the $z$-direction $A_{p}(y, z)=z / a$. For tilted fields $\left(\beta \neq 90^{\circ}\right)$, a circularly polarized probe in the $x z$-plane, ensures that any molecule is ionized and detected with the same probability independently of $\beta$, and it gives $A_{c}(y, z)=1-y^{2} / a^{2}$. The apparent singularity of equation (4) at $y^{2}+z^{2}=a^{2}$ will disappear when we integrate over different ion recoil velocities.

So far we have assumed, that all ions acquire the same recoil velocity. Experimentally, however, they follow a certain distribution $D(a)$, which is assumed to have only nonzero values at positive velocities. The screen image is obtained by averaging over all these velocities with their corresponding weights as

$$
P_{\gamma}^{i}(y, z)=\int_{0}^{\infty} \rho_{\gamma}^{i}(y, z ; a) D(a) d a .
$$

with $i=l, p$ and $c$ depending on the probe polarization. The experimental velocity distribution $D(a)$ for the $\mathrm{CN}^{+}$ ions created in the Coulomb explosion of benzonitrile is shown in Figure 4. The second and third peak are the two Coulomb explosion channels relevant for determining the orientation of the $\mathrm{C}-\mathrm{CN}$ axis. The two peaks are fitted to a combination of two Gaussian functions, after subtraction of background, and used further on in the model. Since $a$ is not the velocity but a distance proportional to it, we have rescaled the abscissa such that the maximum of $D(a)$ is at $a=1$.

In the experiment, the molecular beam contains molecules in different quantum states. A certain level $\gamma=J_{K_{a} K_{c}} M$ has a relative weight $W_{\gamma}=W_{J_{K_{a} K_{c}} M}$ within this ensemble, which is determined by classical trajectory simulations for all relevant rotational states using Monte Carlo sampling of the initial phase distributions [29]. Including the resulting populations in the laser interaction zone, the final 2D-screen probability distribution reads

$$
P_{\mathrm{T}}^{i}(y, z)=\sum_{\gamma} W_{\gamma} P_{\gamma}^{i}(x, y)
$$

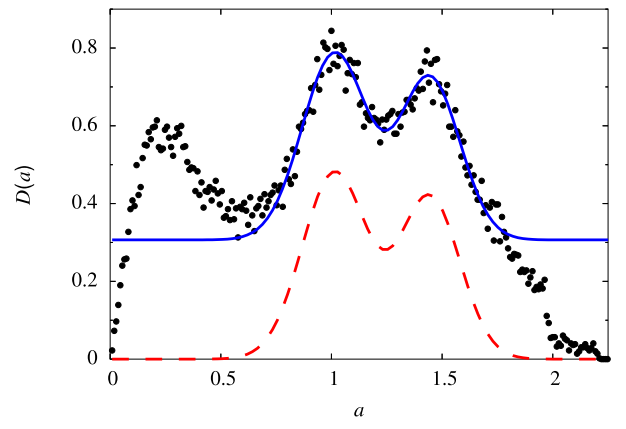

Fig. 4: Experimental recoil velocity distribution of $\mathrm{CN}^{+}$ions rescaled to have a maximum for $a=1$ (points), fitted functions using two Gaussians (solid line) and two Gaussians without background (dashed line).

where the sum runs over all populated states and the index $i$ indicates the polarization of the probe.

\section{The experimental observables}

The alignment of a molecule is quantified by the expectation value $\left\langle\cos ^{2} \theta\right\rangle_{\gamma}$ with $0<\left\langle\cos ^{2} \theta\right\rangle<$ 1 , where larger values correspond to stronger alignment. While $\left\langle\left\langle\cos ^{2} \theta\right\rangle\right\rangle=\sum_{\gamma} W_{\gamma}\left\langle\cos ^{2} \theta\right\rangle_{\gamma}$ is not experimentally determined, the alignment is characterized through $\left\langle\left\langle\cos ^{2} \theta_{2 D}\right\rangle\right\rangle=\sum_{\gamma} W_{\gamma}\left\langle\cos ^{2} \theta_{2 D}\right\rangle_{\gamma}$, where $\theta_{2 D}=\arctan (z / y)$ is the angle between the $z$-axis of the screen plane and the projection of the ion recoil velocity vector onto the detector plane. Let us remark that $\left\langle\cos ^{2} \theta_{2 D}\right\rangle_{\gamma}$ is computed from the final 2D probability density equation (5), whereas $\left\langle\cos ^{2} \theta\right\rangle_{\gamma}$ from the $3 \mathrm{D}$ wave functions.

When the linear polarization of the YAG laser is not perpendicular to the static electric field $\left(\beta \neq 90^{\circ}\right)$, the up/down symmetry of the $2 \mathrm{D}$-images is lost, and an asymmetric distribution appears showing a certain degree of orientation. This up/down asymmetry is experimentally quantified by the ratio $N_{\text {up }} / N_{\text {tot }}$, with $N_{\text {up }}$ being the amount of ions in the upper part of the screen plane, and $N_{\text {tot }}$ the total number of detected ions. In our description, they are given by

$$
N_{u p}=\int_{-\infty}^{\infty} \int_{z \geq 0} P_{\mathrm{T}}(y, z) d y d z,
$$

and

$$
N_{t o t}=\int_{-\infty}^{\infty} \int_{-\infty}^{\infty} P_{\mathrm{T}}(y, z) d y d z
$$

Note that due to the normalization we have that $N_{t o t}=$ 1.

Finally, to compare our numerical data with the experimental results, we take into account the finite spatial width of the YAG and probe pulses. Both laser beams have a Gaussian profile with widths of $\omega_{Y}=36 \mu \mathrm{m}$ and 
$\omega_{P}=21 \mu \mathrm{m}$, respectively, and are overlapped in time and space. The YAG pulse ensures the alignment or orientation of the molecules, whereas the probe pulse is needed for detection purposes. The degree of alignment and the dissociation probability varies with the position of a molecule in the interaction volume. Thus, we integrate over the overlap region of the probe laser with the molecular beam considering the YAG-laser intensity. Hereby, we have assumed a linear behavior of the dissociation efficiency on the probe laser intensity. This is an approximation, as recent works about non resonant strong field dissociation for other systems have proved a $I^{3}$-dependence [46]. However, our calculations indicate that the orientation and alignment results are not very sensitive to a variation of this dependency. We have also assumed that the spatial profile of the molecular beam is much broader than that of the laser beams, hence, the variations of the density of molecules can be neglected.

\section{COMPUTATIONAL RESULTS}

In this section, we apply the above described approach using benzonitrile as a prototype example. Recent experimental results for this molecule [31] allow us to directly compare them to our numerical studies. The moments of inertia are given by $B_{X_{M}}=1214 \mathrm{MHz}, B_{Y_{M}}=1547$ $\mathrm{MHz}$, and $B_{Z_{M}}=5655 \mathrm{MHz}$, the electric dipole moment is $\mu=4.515 \mathrm{D}$, and the principal moments of polarizability are $\alpha_{X_{M} X_{M}}=7.49 \AA^{3}, \alpha_{Y_{M} Y_{M}}=13.01 \AA^{3}$, and $\alpha_{Z_{M} Z_{M}}=18.64 \AA^{3}$ [31, 47].

\section{A. Alignment results}

The experimental fields geometry consists of a weak static electric field perpendicular to the screen with strength $E_{s}=286 \mathrm{~V} \mathrm{~cm}^{-1}$ and a strong alignment laser linearly polarized along the $Z_{L}$-axis ( $z$-axis). For the probe pulse we consider the three possible polarizations: i) linearly polarized perpendicular to the screen; ii) linearly polarized parallel to the screen; and iii) circularly polarized in the $x z$-plane perpendicular to the screen. The computational results shown below include the recoil velocity distribution and the volume effect.

The theoretical results for the density distribution of the molecular beam on the screen are presented in Figure 5(a), (b) and (c) for $E_{S}=286 \mathrm{~V} \mathrm{~cm}^{-1}$ and for YAG pulse intensities $I=5 \times 10^{10} \mathrm{~W} \mathrm{~cm}^{-2}$, $10^{11} \mathrm{~W} \mathrm{~cm}^{-2}$, and $7 \times 10^{11} \mathrm{~W} \mathrm{~cm}^{-2}$, respectively. A strong confinement of the probability distribution is observed, the molecules are aligned with their $Z_{M}$-axis pointing along the polarization axis of the YAG laser. This $1 \mathrm{D}$ alignment becomes more pronounced as $I$ is increased. The two ionization channels that characterize the velocity distribution for BN, cf. Figure 4, appear as double maxima on the upper and lower hump of the $2 \mathrm{D}$ images. They become more prominent as $I$ is increased,
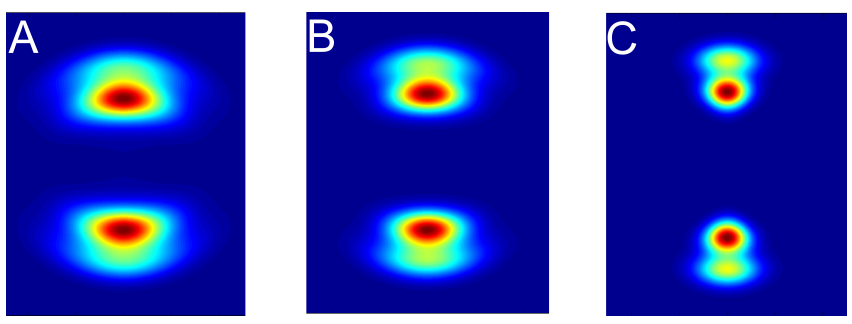

Fig. 5: The 2D projection of the density distribution of the benzonitrile molecular beam for (a) $I=5 \times 10^{10} \mathrm{~W} \mathrm{~cm}^{-2}$, (b) $10^{11} \mathrm{~W} \mathrm{~cm}^{-2}$ and $(\mathrm{c}) 7 \times 10^{11} \mathrm{~W} \mathrm{~cm}^{-2}, E_{S}=286 \mathrm{~V} \mathrm{~cm}^{-1}$ and $\beta=90^{\circ}$.

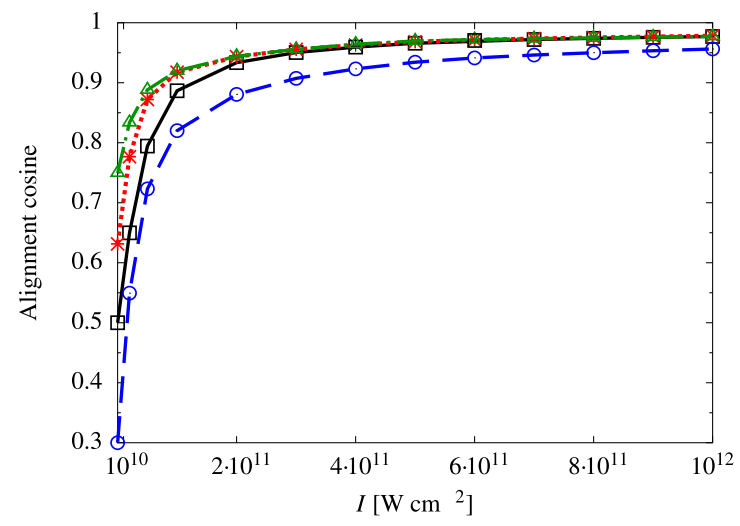

Fig. 6: Alignment cosines for the molecular beam of bezonitrile as a function of the YAG pulse intensity, $E_{S}=286$ $\mathrm{V} \mathrm{cm}{ }^{-1}$ and $\beta=90^{\circ}:\left\langle\left\langle\cos ^{2} \theta\right\rangle\right\rangle$ (circles and dashed line), $\left\langle\left\langle\cos ^{2} \theta_{2 D}\right\rangle\right\rangle^{l}$ (squared and solid line), $\left\langle\left\langle\cos ^{2} \theta_{2 D}\right\rangle\right\rangle^{c}$ (asterisks and dotted line), and $\left\langle\left\langle\cos ^{2} \theta_{2 D}\right\rangle\right\rangle^{p}$ (triangles and dash-dotted line).

i. e., for a YAG pulse with $I=7 \times 10^{11} \mathrm{~W} \mathrm{~cm}^{-2}$, while they are overlapping for lower intensities.

We quantify this alignment by the expectation values $\left\langle\left\langle\cos ^{2} \theta\right\rangle\right\rangle,\left\langle\left\langle\cos ^{2} \theta_{2 D}\right\rangle\right\rangle^{l},\left\langle\left\langle\cos ^{2} \theta_{2 D}\right\rangle\right\rangle^{p}$, and $\left\langle\left\langle\cos ^{2} \theta_{2 D}\right\rangle\right\rangle^{c}$, which are presented in Figure 6 as a function of the YAG intensity $I$ for the whole molecular beam. The indexes $l, p$ and $c$ indicate the probe pulse polarization: linearlypolarized perpendicular and linearly-polarized parallel to the static field and circularly-polarized, respectively. Without the presence of any aligning or orienting fields the screen image is already biased by the "geometric alignment" of the probe pulse. An analytical calculation shows for a random isotropic distribution that $\left\langle\left\langle\cos ^{2} \theta\right\rangle\right\rangle=1 / 3,\left\langle\left\langle\cos ^{2} \theta_{2 D}\right\rangle\right\rangle^{l}=0.5,\left\langle\left\langle\cos ^{2} \theta_{2 D}\right\rangle\right\rangle^{p}=3 / 4$, and $\left\langle\left\langle\cos ^{2} \theta_{2 D}\right\rangle\right\rangle^{c}=5 / 8$. This mild confiment obtained when the probe pulse is circularly polarized or linearly polarized parallel to the screen is due to the enhanced ionization probability for molecules having the $C_{2}$ axis (i. e., the $\mathrm{C}-\mathrm{CN}$ axis) parallel to the laser polarization. If the molecules are exposed only to the weak electric field, $E_{S}=286 \mathrm{~V} \mathrm{~cm}^{-1}$, these field-free values are only slightly perturbed $\left\langle\left\langle\cos ^{2} \theta\right\rangle\right\rangle \approx 0.34,\left\langle\left\langle\cos ^{2} \theta_{2 D}\right\rangle\right\rangle^{l}=0.51$, $\left\langle\left\langle\cos ^{2} \theta_{2 D}\right\rangle\right\rangle^{p}=0.7496$, and $\left\langle\left\langle\cos ^{2} \theta_{2 D}\right\rangle\right\rangle^{c}=0.6319$ [30]. 

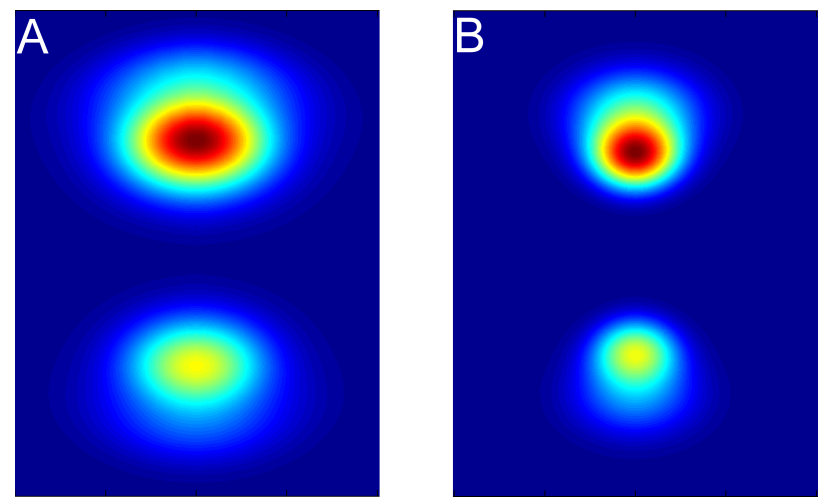

Fig. 7: The 2D projection of the density distribution of the benzonitrile molecular beam for (a) $I=10^{11}$, and (b) $7 \times 10^{11}$ $\mathrm{W} \mathrm{cm}{ }^{-2}, E_{S}=286 \mathrm{~V} \mathrm{~cm}^{-1}$ and $\beta=135^{\circ}$.

These four expectation values qualitatively all show a similar evolution as the YAG pulse intensity is varied: a steep increase followed by a plateau-like behavior which indicates a saturation of the degree of alignment. This dependence of the alignment on the YAG pulse intensity nicely reproduces the experimental behavior observed for a molecular beam of iodobenzene (IB) [15, 29]. Note that the differences due to the polarizations of the probe pulse are noticeable for low intensities, whereas for strong alignment fields they all converge to the same asymptotic limit. For $I=7 \times 10^{11} \mathrm{~W} \mathrm{~cm}^{-2}$, we obtain $\left\langle\left\langle\cos ^{2} \theta\right\rangle\right\rangle=0.946,\left\langle\left\langle\cos ^{2} \theta_{2 D}\right\rangle\right\rangle^{l}=0.972$, $\left\langle\left\langle\cos ^{2} \theta_{2 D}\right\rangle\right\rangle^{p}=0.973$, and $\left\langle\left\langle\cos ^{2} \theta_{2 D}\right\rangle\right\rangle^{c}=0.974$. The value obtained experimentally, 0.89 , for $\left\langle\left\langle\cos \theta_{2 D}\right\rangle\right\rangle^{p}$ [31] is somewhat lower which we ascribe to contaminant ions, like $\mathrm{C}_{2} \mathrm{H}_{2}^{+}$(with the same mass-to-charge ratio as $\mathrm{CN}^{+}$) in the images. Ions like $\mathrm{C}_{2} \mathrm{H}_{2}^{+}$do not have a strong angular confinement and therefore reduced the apparent degree of alignment in the image. For IB, a molecule which is expected to attain an alignment degree similar to that of BN because of a similar polarizabilty tensor, the recoil ion used, $\mathrm{I}^{+}$, is more clean since there are no contaminant ions at the mass of 127 . As a result, the observed degree of alignment is as high as 0.97 for $\left\langle\left\langle\cos \theta_{2 D}\right\rangle\right\rangle^{l}[29]$ in good agreement with the theoretical predictions.

For completeness, we have also considered a thermal sample of $\mathrm{BN}$ at $1 \mathrm{~K}$ and $I=7 \times 10^{11} \mathrm{~W} \mathrm{~cm}^{-2}$, obtaining $\left\langle\left\langle\cos ^{2} \theta_{2 D}\right\rangle\right\rangle^{l}=0.961$, which agrees well with the experimental value for IB [48].

\section{B. Orientation results}

The polarization axis of the YAG-laser is now rotated about the $Y_{L}$-axis, and forms an angle $\beta\left(\beta \neq 90^{\circ}\right)$ with the weak electrostatic field perpendicular to the screen, $E_{s}=286 \mathrm{~V} \mathrm{~cm}^{-1}$. The orientation ratio $N_{u p} / N_{t o t}$ equation (6) is derived for a circularly polarized probe pulse, including the velocity distribution and the volume effect and using the diabatic model described above.
The theoretical results for the projected density distribution of the molecular beam are presented in Figs. 7(a) and (b) for two intensities and $\beta=135^{\circ}$. As a consequence of the rotation of the YAG polarization axis, the up/down symmetries of the wave functions is lost. The 1D orientation becomes more pronounced as $I$ is increased. For $\beta=135^{\circ}$, the projection of $\mathrm{CN}^{+}$ions on the $2 \mathrm{D}$ detector overlaps the two Coulomb explosion channels to an extent that they cannot be discerned.

The theoretical results for the orientation $N_{u p} / N_{t o t}$ are presented in Figure 8 as a function of the tilt angle $\beta$ and for three intensities. The main feature of the $N_{u p} / N_{t o t}$ is that it is almost independent of the alignment laser intensity and of $\beta$. For these three intensities, all the populated states are within the pendular regime. Thus, if the volume effect was neglected, the ratio $N_{u p} / N_{\text {tot }}$ would be independent of $I$ for this pendular regime of laser intensities. By taking into account the spatial distribution of the probe and YAG beams, the molecules show the smallest orientation for $I=10^{11} \mathrm{~W} \mathrm{~cm}^{-2}$. This is explained by the larger contribution of lower intensities, for which the molecules are not yet in the pendular regime, to the volume effect integral for $I=10^{11} \mathrm{~W} \mathrm{~cm}^{-2}$, and as a consequence $N_{u p} / N_{\text {tot }}$ is reduced. For $I=5 \times 10^{11}$ and $7 \times 10^{11} \mathrm{~W} \mathrm{~cm} \mathrm{~cm}^{-2}$, the contribution of lower intensities is not strong enough to cause any noticeable difference. Note that for IB, it was also experimentally found that $N_{u p} / N_{\text {tot }}$ is independent of the YAG pulse intensity [29]. For $\beta=90^{\circ}$, the molecular beam does not show any

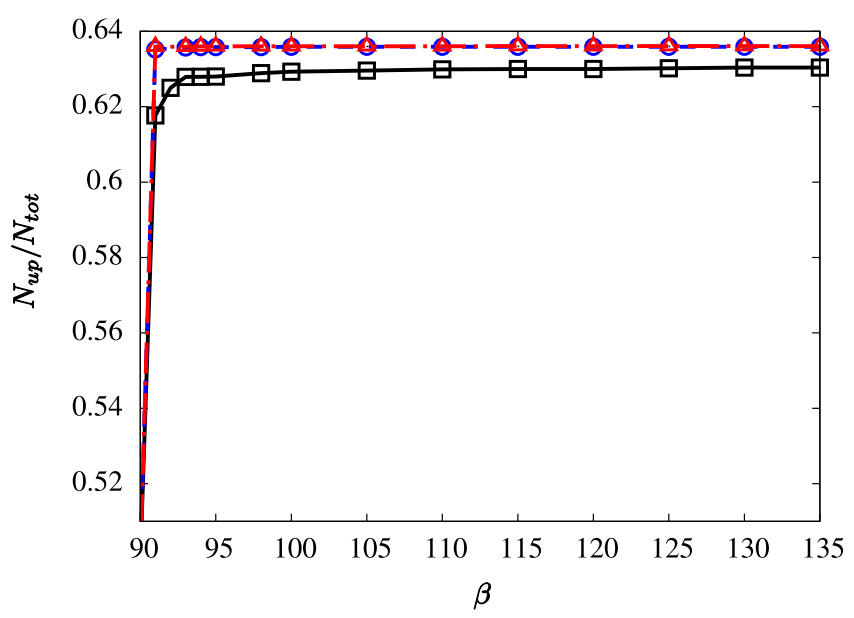

Fig. 8: The theoretical orientation ratio $N_{u p} / N_{t o t}$ as a function of the angle $\beta$ for the molecular beam of benzonitrile, YAG pulses with $I=10^{11}$ (squares and solid line), $5 \times 10^{11}$ (circles and dashed line) and $7 \times 10^{11} \mathrm{~W} \mathrm{~cm}^{-2}$ (triangles and dash-dotted line) and a static field with $E_{S}=286 \mathrm{~V} \mathrm{~cm}^{-1}$.

orientation and it holds $N_{u p} / N_{\text {tot }}=0.5$. For a certain YAG pulse intensity, $N_{u p} / N_{\text {tot }}$ quickly increases as $\beta$ is increased from 90 and reaches a plateau-like behavior for the rest of the field configurations. For $\beta=135^{\circ}$ and $I=$ $7 \times 10^{11} \mathrm{~W} \mathrm{~cm}^{-2}$, we obtain $N_{u p} / N_{\text {tot }}=0.636$ which is smaller than the experimental result of 0.71 [31]. Thus, 
our theoretical model underestimates the orientation obtained in the experiment.

An important discrepancy between theoretical and experimental results is the variation of $N_{u p} / N_{t o t}$ for $\beta$ close to $90^{\circ}$. For a molecular beam of IB [29], it was experimentally shown that $N_{u p} / N_{\text {tot }}$ smoothly increases (decreases) as $\beta$ is increased (decreased) from $90^{\circ}$, reaching for $\beta \lesssim 60^{\circ}$ and $\beta \gtrsim 120^{\circ}$ the plateau. In contrast, the theoretical $N_{u p} / N_{\text {tot }}$ sharply increases, and its $\beta$ independent value is already achieved for $\beta \gtrsim 91^{\circ}$. In the pendular regime, the strong laser interaction pairs states into quasidegenerate doublets. The electrostatic field can induce a strong coupling between these levels giving rise to a large orientation if the energy gap is small enough. For certain intensities, some excited levels may not show such a narrow energy gap to obtain a significant orientation, but, their relative weight within the molecular beam is so small that their contribution to the final result is not relevant. For a linear molecule in combined fields [49], such a sharp rise was predicted for the expectation value $\langle\cos \theta\rangle$ as the static field strength is increased, i.e., increasing or decreasing $\beta$ from $90^{\circ}$ in our case. On Figure 2 of Friedrich and Herschbach Ref. 22 this effect is obtained for the interaction with the laser field being 25000 times larger than the one with the static field. We could perform a similar comparison and for $\mathrm{BN}, I=7 \times 10^{11}$ $\mathrm{W} \mathrm{cm}{ }^{-2}, E_{S}=286 \mathrm{~V} \mathrm{~cm}^{-1}$ and $\beta=95^{\circ}$, the interaction of the YAG pulse, $2 \pi I \alpha^{Z X} / c$, is 43600 times larger than the coupling with the static field, $\mu E_{S} \cos \beta$.

Let us remark, that our diabatic model for the population transfer is equivalent to considering a field configuration with a linearly polarized YAG pulse being parallel to an electrostatic field with strength $E_{s} \cos \beta$. This field geometry is neglecting the component of the electric field $E_{s} \sin \beta$ perpendicular to the YAG polarization, which is responsible for breaking the azimuthal symmetry and causing $M$ to stop being a good quantum number. This approximation can be done because the interaction due to the static field is sufficiently weak. We have performed the full calculation considering the simplified case of parallel fields, the static one with strength $E_{s}=286 \cos 135^{\circ}$ $\mathrm{V} \mathrm{cm}^{-1}$ and $I=7 \times 10^{11} \mathrm{~W} \mathrm{~cm}^{-2}$, obtaining for the orientation the value $N_{u p} / N_{\text {tot }}=0.636$.

To illustrate the necessity of the diabatic model, we build up the molecular ensemble by successively adding states according to their weight. Thus, our initial ensemble contains only the ground state, and for the second one, we add the second and third most populated levels. The relative weights of the states within these molecular beams are the same as in the full molecular ensemble. Figure 9 presents the orientation $N_{u p} / N_{\text {tot }}$ as a function of the percentage of states included in the ensemble for $I=7 \times 10^{11} \mathrm{~W} \mathrm{~cm}^{-2}$ and $\beta=135^{\circ}$. The ratio $N_{u p} / N_{\text {tot }}$ has been computed with the diabatic model for the population transfer and a fully adiabatic picture, i.e., all avoided crossings are assumed to be passed adiabatically. The first three populated states, $0_{00} 0,1_{01} 1$ and $1_{01}-1$, are perfectly oriented: $N_{u p} / N_{t o t}=1$. For the sets

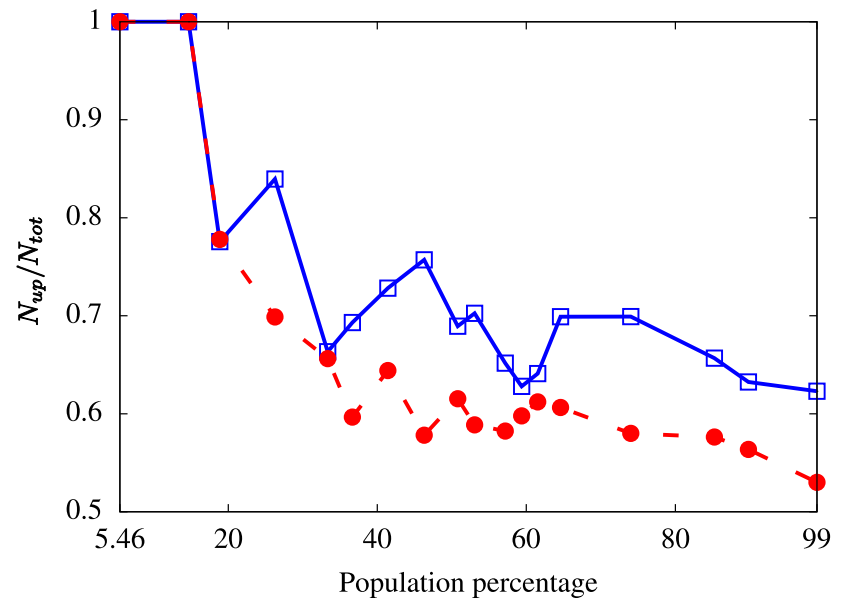

Fig. 9: The theoretical orientation ratio $N_{u p} / N_{\text {tot }}$ as a function of the population of the molecular beam of benzonitrile, for the field configuration $I=7 \times 10^{11} \mathrm{~W} \mathrm{~cm}{ }^{-2}$, $E_{S}=286 \mathrm{~V} \mathrm{~cm}^{-1}$, and $\beta=135^{\circ}$, computed with diabatic model for the population transfer (squares and solid line) and a fully adiabatic picture (circles and dashed line).

including the levels adding $20 \%$ of the total population, the diabatic and adiabatic results agree because these states do not suffer any avoided crossings. As highly excited levels are added to the molecular beam, the difference between both calculations becomes more evident due to the presence of the diabatic avoided crossings. They both show a zig-zag decreasing trend as the population is increased. The ratio $N_{u p} / N_{\text {tot }}$ computed under the adiabaticity assumption is smaller than the diabatic result as more states are added. Taking into account all states populated in the molecular beam, the adiabatic model does not yield any appreciable amount of orientation: we obtain $N_{u p} / N_{\text {tot }}=0.53$ which strongly underestimates the experimental result. In addition we have observed that the adiabatic orientation ratio $N_{u p} / N_{\text {tot }}$ is no longer $\beta$-independent. This confirms that non-adiabatic crossings play a crucial role for understanding the strong orientation observed in the experiment.

Finally, it is worth noting for the field configuration $I=7 \times 10^{11} \mathrm{~W} \mathrm{~cm}^{-2}$ and $\beta=135^{\circ}$, a thermal sample of $\mathrm{BN}$ at $1 \mathrm{~K}$ shows a weak degree of orientation with $N_{u p} / N_{\text {tot }}=0.563$.

\section{SUMMARY AND CONCLUSIONS}

We have presented a theoretical model to investigate the degree of alignment and orientation of a beam of asymmetric top molecules exposed to combined electrostatic and non-resonant linearly polarized laser fields. Our analysis combines the field-dressed wave functions with the experimental distribution of the populated quantum states. As a first step, we solve the threedimensional Schrödinger equation within the rigid rotor approximation. For a certain field geometry, we 
treat each irreducible representation independently, by expanding the wave function in a basis that respects the corresponding symmetries. Since the dc electric field strength is very weak, we consider $M$ as being almost conserved, and a diabatic model is introduced to approximate the population transfer trough the avoided crossings as the YAG pulse intensity is increased. The 2D projection of a wave function is derived by using the detection selectivity factors of the probe pulse, the velocity distribution of the detected ions, and a volume effect average.

This theoretical model has been checked by comparing the numerical and experimental results for benzonitrile. The molecular beam has been simulated using the population of each quantum state numerically obtained from deflection profiles 29]. For several field configurations, we have performed a detailed study of the directional properties of the molecular mixture. In particular, we have explored the degrees of alignment and orientation as the YAG pulse intensity and the angle between both fields are varied. For perpendicular fields, a good agreement between the computational and experimental results is obtained. Let us remark that we do not take into account the background of unwanted ions which contaminate the velocity mapping images for $\mathrm{BN}$ and reduce the experimental degree of alignment [31]. Hence, a better agreement could be achieved for other systems with cleaner Coulomb explosion imaging channels, like IB.

Regarding the orientation results, we have shown that the assumption of a fully adiabatic dynamics is incorrect for the prototypical experiment and predicts a nonoriented molecular beam - which is not in agreement with the experimental results. Indeed, we have proven that the degree of orientation does not adiabatically follow the time envelope of the YAG laser with a $\mathrm{FWHM}=10$ $\mathrm{ns}$ in the experiment. By employing a simple diabatic model, the experimental results for orientation could be reproduced reasonably well. Based on the comparison with the experimental measures, the important impact of the diabaticity on the field-dressed molecular dynamics is hereby demonstrated. However, notice that our model does not produce the smooth $\beta$-dependence of $N_{u p} / N_{t o t}$ that was experimentally obtained for IB [29].

This theoretical model is based on several approximations that may be the source of discrepancies with the experimental results. The molecules are exposed to an alignment laser pulse with a certain time profile, but we are performing a time-independent description of the field-dressed rotational dynamics. We compensate this deficiency by using a simple partially diabatic approach to model the populations transfer. However, a general statement on the character of the avoided crossings can not be made, and some of them could not be classified clearly by our adiabaticity criterion. The semiclassical calculations of the molecular trajectories through the deflector have been derived using a fully adiabatic picture, whereas it has been theoretically shown that such an approximation may be incorrect 39]. These assumptions for both processes might not be fully satisfied. A full time-dependent description of the process would properly treat the avoided crossing, but it is computationally very challenging. Possible distortion effects due to the strong laser pulse are also not taken into account. Furthermore, we are working within the axial recoil approximation neglecting the interactions between the ionic fragments on their way to the detector. Analogously, collisions and interactions between the molecules within the molecular beam [50] were not considered. The geometric alignment due to the strong-field ionization has been assumed to be a single-photon absorption, but it is a multiphoton process [46]. Nuclear hyperfine structure has been neglected. Whereas, it can be of the same order of magnitude as the interaction with the weak static field (i.e., for IB [51]), this is clearly not the case for BN [47].

Certainly, it would be interesting to perform a comparison of these computational results with experimental data obtained for other molecular species either with a smaller number of populated states or with a cleaner imaging signal. A rather natural extension of the present work would be to go beyond any of the approximations described above. A more sensitive criterion for the avoided crossing treatment, a time-dependent description, or the inclusion of the hyperfine interaction should improve these theoretical results.

\section{Acknowledgments}

We acknowledge support by Frank Filsinger for the trajectory simulations providing the populations of individual quantum states. We thank Gerard Meijer for continuous interest and support of this project and Marko Härtelt and Bretislav Friedrich for helpful discussions. Financial support by the Spanish project FIS2008-02380 (MICINN) as well as the Grants FQM-2445 and FQM4643 (Junta de Andalucía) is gratefully appreciated. J.J.O. acknowledges the support of ME under the program FPU. R.G.F. and J.J.O. belong to the Andalusian research group FQM-207.
1 P. R. Brooks and E. M. Jones, J. Chem. Phys. 45, 3449 (1966).

2 S. Stolte, Ber. Bunsen. Phys. Chem. 86, 413 (1982).

3 R. Zare, Science 20, 1875 (1998).

4 V. Aquilanti, M. Bartolomei, F. Pirani, D. Cappelletti, and F. Vecchiocattivi, Phys. Chem. Chem. Phys 7, 291 (2005).

5 A. Landers, T. Weber, I. Ali, A. Cassimi, M. Hattass, O. Jagutzki, A. Nauert, T. Osipov, A. Staudte, M. Prior, et al., Phys. Rev. Lett. 87, 013002 (2001). 
6 L. Holmegaard, J. L. Hansen, L. Kalhoj, S. L. Kragh, H. Stapelfeldt, F. Filsinger, J. Küpper, G. Meijer, D. Dimitrovski, M. Abu-samha, et al., Nat. Phys. 6, 428 (2010).

7 C. Z. Bisgaard, O. J. Clarkin, G. R. Wu, A. M. D. Lee, O. Gessner, C. C. Hayden, and A. Stolow, Science 323, 1464 (2009).

8 J. Levesque, Y. Mairesse, N. Dudovich, H. Pépin, J.-C. Kieffer, P. B. Corkum, and D. M. Villeneuve, Phys. Rev. Lett. 99, 243001 (2007).

9 R. Velotta, N. Hay, M. B. Mason, M. Castillejo, and J. P. Marangos, Phys. Rev. Lett. 87, 183901 (2001).

10 J. C. H. Spence and R. B. Doak, Phys. Rev. Lett. 92, 198102 (2004).

11 F. Filsinger, G. Meijer, H. Stapelfeldt, H. Chapman, and J. Küpper, Phys. Chem. Chem. Phys. 13, 2076 (2011).

12 R. J. D. Miller, R. Ernstorfer, M. Harb, M. Gao, C. T. Hebeisen, H. Jean-Ruel, C. Lu, G. Moriena, and G. Sciaini, Acta Crystallogr. A 66, 137 (2010).

13 S. Y. T. van de Meerakker, H. L. Bethlem, and G. Meijer, Nature Phys. 4, 595 (2008).

14 H. Stapelfeldt and T. Seideman, Rev. Mod. Phys. 75, 543 (2003).

15 L. Holmegaard, J. H. Nielsen, I. Nevo, H. Stapelfeldt, F. Filsinger, J. Küpper, and G. Meijer, Phys. Rev. Lett. 102, 023001 (2009).

16 O. Ghafur, A. Rouzée, A. Gijsbertsen, W. K. Siu, S. Stolte, and M. J. J. Vrakking, Nature Phys. 5, 289 (2009).

17 A. Rouzée, A. Gijsbertsen, O. Ghafur, O. M. Shir, T. Bäck, S. Stolte, and M. J. J. Vrakking, New J. Phys. 11, 105040 (2009).

18 M. H. G. de Miranda, A. Chotia, B. Neyenhuis, D. Wang, G. Quéméner, S. Ospelkaus, J. L. Bohn, J. Ye, and D. S. Jin, Nature Physics (2011).

19 J. Reuss, in Atomic and molecular beam methods, edited by G. Scoles (Oxford University Press, New York, NY, USA, 1988), vol. 1, chap. 11, p. 276, ISBN 0195042808.

20 H. J. Loesch and A. Remscheid, J. Chem. Phys. 93, 4779 (1990).

21 B. Friedrich and D. R. Herschbach, Z. Phys. D 18, 153 (1991).

22 B. Friedrich and D. R. Herschbach, J. Chem. Phys. 111, 6157 (1999).

23 B. Friedrich and D. R. Herschbach, J. Phys. Chem. A 103, 10280 (1999).

24 R. Baumfalk, N. H. Nahler, and U. Buck, J. Chem. Phys. 114, 4755 (2001).

25 H. Sakai, S. Minemoto, H. Nanjo, H. Tanji, and T. Suzuki, Phys. Rev. Lett. 90, 083001 (2003).

26 H. Tanji, S. Minemoto, and H. Sakai, Phys. Rev. A 72, 063401 (2005).

27 M. Schnell and G. Meijer, Angew Chem Int Edit 48, 6010 (2009).

28 J. Küpper, F. Filsinger, and G. Meijer, Faraday Disc. 142, 155 (2009).

29 F. Filsinger, J. Küpper, G. Meijer, L. Holmegaard, J. H.
Nielsen, I. Nevo, J. L. Hansen, and H. Stapelfeldt, J. Chem. Phys. 131, 064309 (2009).

30 I. Nevo, L. Holmegaard, J. H. Nielsen, J. L. Hansen, H. Stapelfeldt, F. Filsinger, G. Meijer, and J. Küpper, Phys. Chem. Chem. Phys. 11, 9912 (2009).

31 J. L. Hansen, L. Holmegaard, L. Kalhøj, S. L. Kragh, H. Stapelfeldt, F. Filsinger, G. Meijer, J. Küpper, D. Dimitrovski, M. Abu-samha, et al., Phys. Rev. A 83, 023406 (2011).

32 M. Härtelt and B. Friedrich, J. Chem. Phys. 128, 224313 (2008).

33 J. J. Omiste, R. González-Férez, and P. Schmelcher (2011), in preparation

34 C. A. Arango and G. S. Ezra, Int. J. Bif. and Chaos 18, 1127 (2008).

35 C. A. Arango, W. W. Kennerly, and G. S. Ezra, J. Chem. Phys. 122, 184303 (2005).

36 I. N. Kozin and R. N. Roberts, J. Chem. Phys. 118, 10523 (2003).

37 R. N. Zare, Angular momentum: understanding spatial aspects in chemistry and physics (New York: John Wiley and Sons, 1988).

38 R. Kanya and Y. Ohshima, Phys. Rev. A 70, 013403 (2004).

39 R. Escribano, B. Maté, F. Ortigoso, and J. Ortigoso, Phys. Rev. A 62, 023407 (2000).

40 G. W. King, R. M. Hainer, and P. C. Cross, J. Chem. Phys. 11, 27 (1943).

41 J. Bulthuis, J. Miller, and H. J. Loesch, J. Phys. Chem. A 101, 7684 (1997).

42 W. Kong and J. Bulthuis, J. Phys. Chem. A 104, 1055 (2000).

43 A. Schwettman, J. Franklin, K. R. Overstreet, and J. P. Shaffer, J. Chem. Phys. 123, 194305 (2005).

44 D. Bohm, Quantum Theory (Dover Publications, Inc., New York, 1989), chap. 20.

45 R. Kanya and Y. Ohshima, J. Chem. Phys. 121, 9489 (2004).

46 J. H. Nielsen, P. Simesen, C. Z. Bisgaard, H. Stapelfeldt, F. Filsinger, B. Friedrich, G. Meijer, and J. Küpper, Phys. Chem. Chem. Phys. p. submitted to special issue "physics and chemistry of cold molecules" (2011).

47 K. Wohlfart, M. Schnell, J.-U. Grabow, and J. Küpper, J. Mol. Spec. 247, 119 (2008).

48 V. Kumarappan, C. Z. Bisgaard, S. S. Viftrup, L. Holmegaard, and H. Stapelfeldt, J. Chem. Phys. 125, 194309 (pages 7) (2006).

49 B. Friedrich and D. R. Herschbach, Z. Phys. D 36, 221 (1995).

50 U. Erlekam, M. Frankowski, G. von Helden, and G. Meijer, Phys. Chem. Chem. Phys. 9, 3786 (2007).

51 O. Dorosh, E. Białkowska-Jaworska, Z. Kisiel, and L. Pszczółkowski, J. Mol. Spec. 246, 228 (2007). 